\title{
Neutron monitor count rate increase as a proxy for dose rate assessment at aviation altitudes during GLEs
}

\author{
Alexander Mishev ${ }^{1,2, *}$, Sasu Tuohino ${ }^{3}$, and Ilya Usoskin ${ }^{1,2}$ \\ ${ }^{1}$ Space Climate Research Unit, University of Oulu, 90570 Oulu, Finland \\ 2 Sodankylä Geophysical Observatory, University of Oulu, 90570 Oulu, Finland \\ ${ }^{3}$ Faculty of Science, University of Oulu, 90570 Oulu, Finland
}

Received 14 December 2017 / Accepted 13 August 2018

\begin{abstract}
Radiation exposure due to cosmic rays, specifically at cruising aviation altitudes, is an important topic in the field of space weather. While the effect of galactic cosmic rays can be easily assessed on the basis of recent models, estimate of the dose rate during strong solar particle events is rather complicated and time consuming. Here we compute the maximum effective dose rates at a typical commercial flight altitude of $35 \mathrm{kft}(\approx 11000 \mathrm{~m}$ above sea level) during ground level enhancement events, where the necessary information, namely derived energy/rigidity spectra of solar energetic particles, is available. The computations are carried out using different reconstructions of the solar proton spectra, available in bibliographic sources, leading to multiple results for some events. The computations were performed employing a recent model for effective dose and/or ambient dose equivalent due to cosmic ray particles. A conservative approach for the computation was assumed. A highly significant correlation between the maximum effective dose rate and peak NM count rate increase during ground level enhancement events is derived. Hence, we propose to use the peak NM count rate increase as a proxy in order to assess the peak effective dose rate at flight altitude during strong solar particle events using the real time records of the worldwide global neutron monitor network.
\end{abstract}

Keywords: Solar energetic particles / GLE events / Neutron monitor network / Radiation environment

\section{Introduction}

According to the generally accepted definition, space weather concerns dynamic conditions on the Sun and solar wind resulting in changes in the Earth's radiation environment, magnetosphere and ionosphere, which eventually can compromise the performance of spacecraft and ground-based systems and can endanger human health (e.g. Baker, 1998; Lilensten \& Bornarel, 2009).

Active processes on the Sun, such as Coronal Mass Ejections (CMEs), solar flares and high-speed solar wind streams may lead to sequence(s) of disturbances in the Earth's magnetosphere and atmosphere, occasionally even impacting the ground level as geomagnetic storms and enhancements of relativistic electron populations in outer radiation belts. Trapped protons and ions can damage satellites. An important part of space weather effects is related to the variable and highly dynamic radiation environment in the near-Earth space and Earth's atmosphere (e.g. Vainio et al., 2009, and references

\footnotetext{
*Corresponding author: alex_mishev@yahoo.com
}

therein). Sporadically, following such solar eruptive processes as solar flares and CMEs Solar Energetic Particles (SEPs) can be produced (e.g. Reames, 1999; Cliver et al., 2004; Reames, 2013; Desai \& Giacalone, 2016; Klein \& Dalla, 2017, and references therein). SEP events are distinct enhancements of particle fluxes originating from Sun.

In this work we focus on solar energetic protons. Their energy is usually of the order of a few tens of $\mathrm{MeV} /$ nucleon, but can occasionally reach about hundred $\mathrm{MeV} /$ nucleon or even above a $\mathrm{GeV} /$ nucleon. While less energetic SEPs are fully absorbed in the atmosphere, more energetic ones can initiate an atmospheric cascade, similarly to Galactic Cosmic Rays (GCRs), whose secondaries eventually reach the ground, leading to an enhancement of count rates of ground based detectors, in particular Neutron Monitors (NMs). This special class of SEP events is known as Ground Level Enhancements (GLEs). Their occurrence rate is about 10-12 per solar cycle with a higher probability during maximum and decline phase of the solar activity cycle (Shea \& Smart, 1990; Stoker, 1995). The high energy SEPs can dramatically change the Earth's radiation environment (Matthiä et al., 2009a; Vainio 
et al., 2009; Sato et al., 2014). Therefore, strong SEP events form a potential space weather hazard, specifically at aviation altitudes.

The increased intensity of secondary cosmic rays at flight altitudes, specifically during SEP events, is an important space weather issue (e.g. Mewaldt, 2006; Pulkkinen, 2007; Shea \& Smart, 2012, and references therein). Aircrews are exposed to an additional complex radiation field, particularly during intercontinental flights over the sub-polar and polar regions, where the magnetospheric shielding is marginal. Recently it was advised to consider the exposure to cosmic radiation of aircrew as occupational (ICRP, 2007). Accordingly, a health monitoring and assessment of the individual accumulated doses of the flight personnel was suggested in the EU (EURATOM, 2013).

The radiation environment, accordingly aircrew exposure depends on geographic position, altitude and solar activity (Spurny et al., 1996, 2003; Shea \& Smart, 2000) and is mainly defined by GCRs, which originate from the Galaxy and consist mostly of protons and $\alpha$-particles with small abundance of heavier nuclei (e.g. Grieder, 2001; Gaisser \& Stanev, 2010, and references therein). The GCR flux, specifically the lowenergy part, is modulated by the solar magnetic field in the Heliosphere inversely following the 11-year solar cycle (e.g. Dorman, 2006; Potgieter, 2013, and references therein). When cosmic ray particles penetrate into the atmosphere, they induce a complicated nuclear-electromagnetic-muon cascade that ionizes the ambient air (Bazilevskaya et al., 2008; Usoskin et al., 2009) and results in enhancement of radiation exposure compared to the sea level (Shea \& Smart, 2000; Vainio et al., 2009).

The complex radiation environment at flight altitudes is governed by GCRs. It is determined by different types and energy ranges of the produced secondary particles. On the other hand, high energy SEP events may enhance the radiation exposure in the atmosphere at commercial flight altitudes, specifically in the polar regions (e.g. Shea \& Smart, 2000; Spurny et al., 2003). During strong GLE events the radiation exposure is a superposition of the GCRs and SEPs contributions. In addition, the characteristics of SEPs, which determine the radiation environment during GLEs: spectra, angular distribution and duration, differ from event to event (Gopalswamy et al., 2012; Moraal \& McCracken, 2012). Therefore, the radiation exposure during GLE events is usually assessed retrospectively using reconstructed spectra from ground based and/or space-borne instruments, individually for each event.

Several space weather issues can be addressed using spaceborne measurements and thorough modelling using improved models of solar activity, interaction of the solar wind with the magnetosphere and models of the radiation belts (e.g. Eastwood et al., 2017; Ganushkina et al., 2017; Koskinen et al., 2017, and references therein). However, assessments of the radiation hazard, specifically during strong SEPs events, are really challenging since it requires detailed information of SEP spectral and anisotropy characteristics. The low energy part of SEP characteristics can be estimated using space-borne particle measurements (e.g. Aguilar et al., 2010; Adriani et al., 2016), accordingly the higher energy part, namely GLE particles, can be studied by NMs (e.g. Dorman, 2004, and references therein). Unfortunately, estimates of the GLE spectral and angular characteristics using measurements from the global
NM network require accurate modelling of their propagation through the Earth's atmosphere and magnetosphere, which is time consuming (e.g. Shea \& Smart, 1982; Humble et al., 1991; Cramp et al., 1997; Mishev \& Usoskin, 2013). Besides, possible systematic errors of the retrieved spectral and angular characteristics of SEPs can lead to significant uncertainties of the estimated radiation exposure (Bütikofer \& Flückiger, 2013, 2015).

In this work, we perform a statistical analysis of 34 GLEs out of 72 registered, where the information about the spectral characteristics is available (e.g. Tuohino et al., 2018). For several events there exist different reconstructions of the solar proton spectra. Therefore, for those events we employ various spectra leading to several results for one event. We compute conservatively the maximal effective dose rate during those evens employing a recently proposed model and the derived GLE spectra. As a result we propose a convenient proxy for the maximum effective dose rate due to SEPs at commercial aviation altitudes during GLEs, which is suitable for operational purposes.

\section{Global neutron monitor network}

Neutron monitors are the main detectors for continuous recording of CR intensity variations (e.g. Moraal, 1976; Debrunner et al., 1988; Lockwood et al., 1990b; Gil et al., 2015; Kudela, 2016). Besides, NMs records are also used to derive the spectral and angular characteristics of GLE and high-energy SEP particles (e.g. Shea \& Smart, 1982; Cramp et al., 1997; Bombardieri et al., 2006; Vashenyuk et al., 2006; Mishev et al., 2014b, 2017). The NM was invented for the International Geophysical Year $1957-1958$ as the IGY neutron monitor (Simpson et al., 1953; Simpson, 1957). Its design was improved leading to the standard detector known as NM64 (Carmichael, 1968; Hatton, 1971; Stoker et al., 2000). The global NM network presently consists of about 50 stations spread over the world (Fig. 1) (Moraal et al., 2000; Mavromichalaki et al., 2011).

The sensitivity of NMs to primary CR is governed by both geomagnetic and atmospheric shielding, leading to the effective cut-off, which is determined by the geomagnetic location and the altitude above sea level. The latter determines the thickness of atmospheric layer above the monitor, since the primary CR must possess a minimum energy necessary to induce an atmospheric shower $(\approx 430 \mathrm{MeV} / \mathrm{n}$ at the sea level), which can reach the ground (e.g. Grieder, 2001; Dorman, 2004). The geomagnetic cut-off rigidity is marginal in the polar regions, where the atmospheric cut-off dominates the shielding. Therefore, polar NMs, particularly high-altitude ones such as SOPO/SOPB and DOMC/DOMB (Fig. 1 and Table 1) are more sensitive to primary CR, specifically SEPs, than mid and highcut-off rigidity NMs. In addition, polar NMs possess a better angular resolution, which is important for the GLE analysis (Bieber \& Evenson, 1995). An illustration of the asymptotic directions at quiet magnetospheric conditions for several low cut-off rigidity NMs is shown in Figure 2. The asymptotic directions are plotted in the range of maximal response of NMs to GLE particles. 


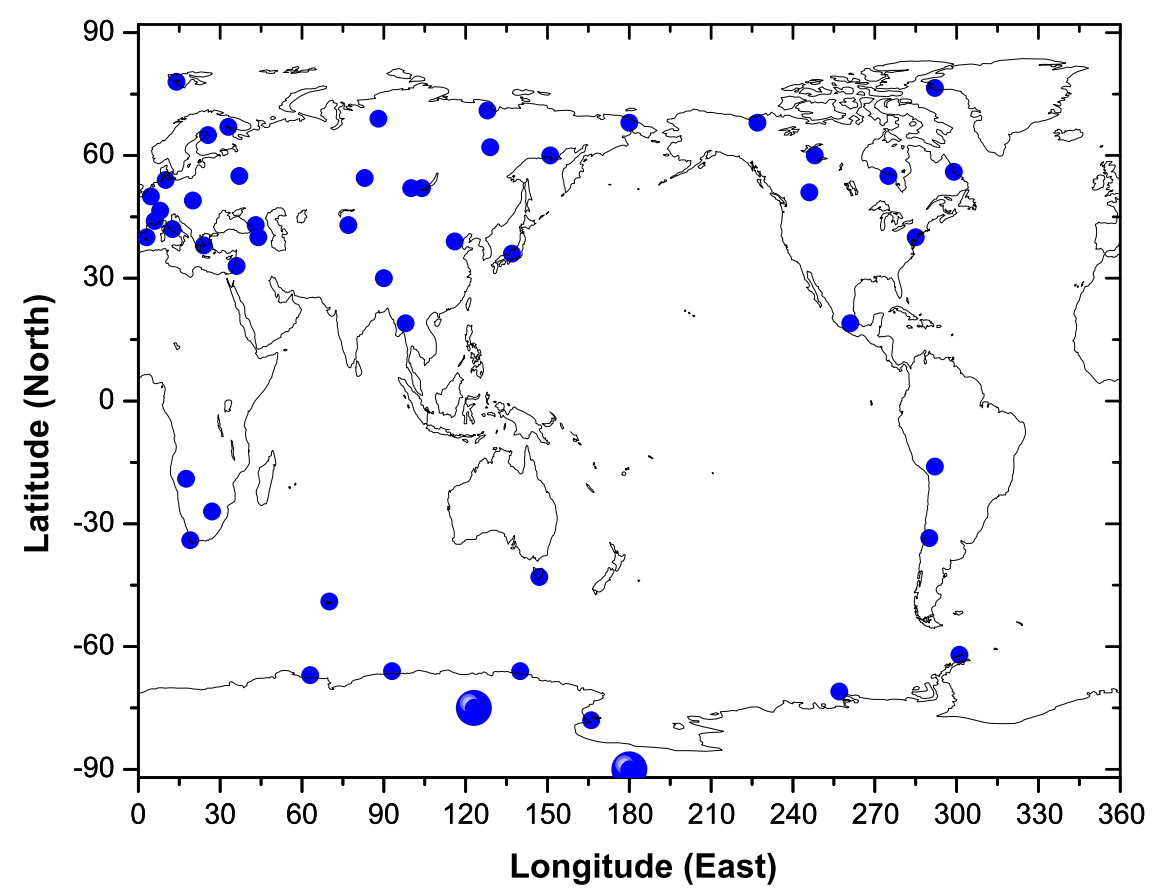

Fig. 1. Current status of the global neutron monitor network. Blue circles depict the NM stations. Bigger circles denote the two high-altitude polar NM stations at Dome C $\left(75^{\circ} \mathrm{S}, 123^{\circ} \mathrm{E}\right)$ and South Pole $\left(90^{\circ} \mathrm{S}\right)$.

Table 1. Low-rigidity and polar neutron monitors with the corresponding geomagnetic cut-off rigidities vs. 2015 and altitudes above sea level.

\begin{tabular}{|c|c|c|c|c|}
\hline Station & Latitude [deg] & Longitude [deg] & $P_{\mathrm{c}}[\mathrm{GV}]$ & Altitude $[\mathrm{m}]$ \\
\hline Barentsburg (BRBG) & 78.03 & 14.13 & 0.01 & 51 \\
\hline Cape Schmidt (CAPS) & 68.92 & 180.53 & 0.45 & 0 \\
\hline Dome C (DOMC) & -75.06 & 123.20 & 0.01 & 3233 \\
\hline Forth Smith (FSMT) & 60.02 & 248.07 & 0.381 & 0 \\
\hline Inuvik (INVK) & 68.35 & 226.28 & 0.16 & 21 \\
\hline Mawson (MWSN) & -67.6 & 62.88 & 0.22 & 0 \\
\hline Mirny (MRNY) & -66.55 & 93.02 & 0.03 & 30 \\
\hline Nain (NAIN) & 56.55 & 298.32 & 0.28 & 0 \\
\hline Neumayer (NEUM) & -70.40 & 351.04 & 0.85 & 0 \\
\hline Norilsk (NRLK) & 69.26 & 88.05 & 0.52 & 0 \\
\hline Oulu (OULU) & 65.05 & 25.47 & 0.69 & 15 \\
\hline Thule (THUL) & 76.60 & 291.2 & 0.1 & 260 \\
\hline Tixie (TXBY) & 71.60 & 128.90 & 0.53 & 0 \\
\hline
\end{tabular}

For the computations of the NM cut-off rigidity and asymptotic directions we employed the MAGNETOCOSMICS code (Desorgher et al., 2005). We used a combination of the internal geomagnetic model IGRF (epoch 2015) (Macmillan et al., 2003) and the external Tsyganenko-89 model (Tsyganenko, 1989), which offers a good balance between simplicity and precision (Kudela \& Usoskin, 2004; Nevalainen et al., 2013).

\section{Model for dose rate computation at flight altitude}

In order to estimate the radiation exposure at flight altitudes one needs precise information of the spectral and angular parameters of energetic particles and to posses a precise model for their propagation in the atmosphere. A convenient way to 


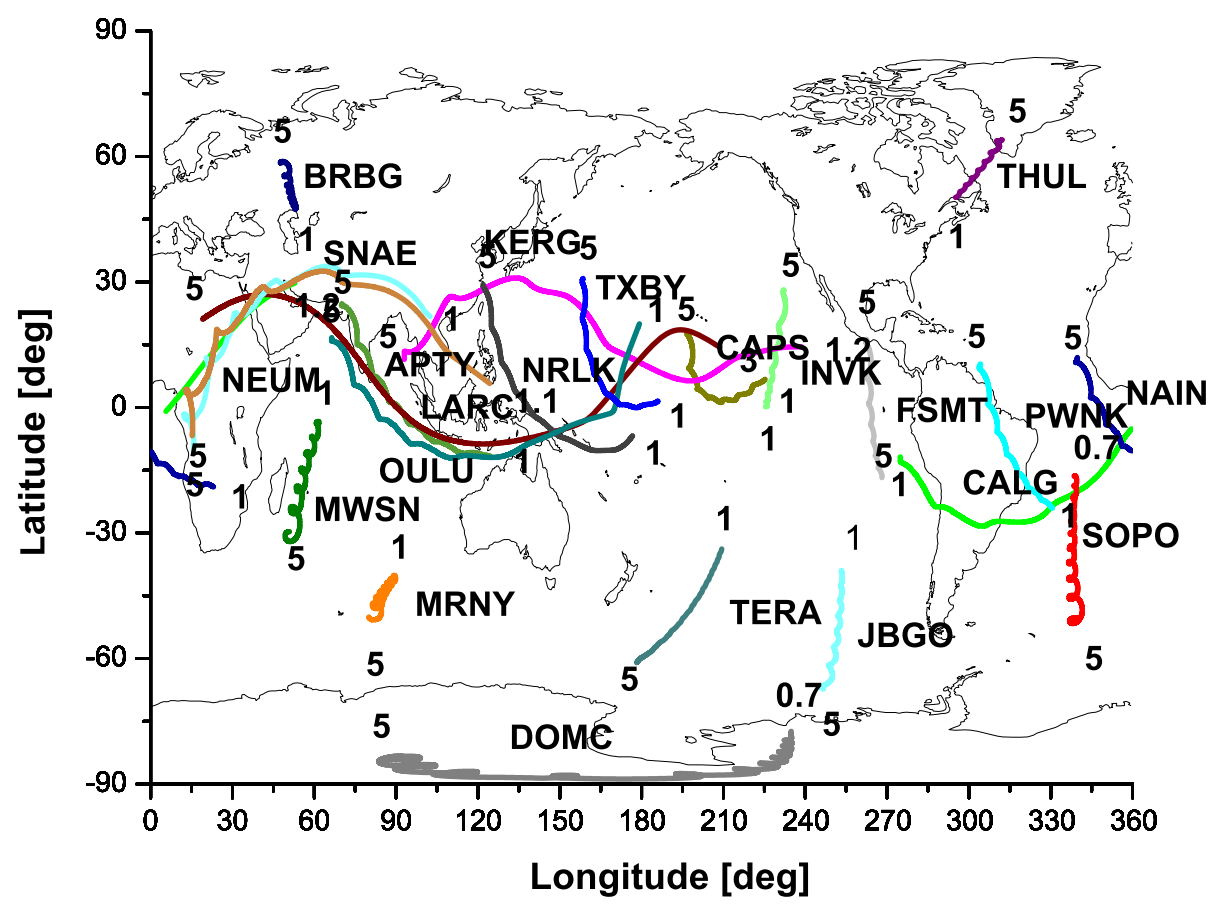

Fig. 2. Asymptotic directions of polar NMs computed for the epoch 2015 at quiet magnetospheriC conditions. The abbreviations are given in Table 1. The color lines depict asymptotic directions plotted in the rigidity range $1-5 \mathrm{GV}$, for DOMC and SOPO from 0.7 to $5 \mathrm{GV}$ respectively.

compute the radiation exposure at a typical flight altitude is based on a Monte Carlo simulation of CR particles propagation and interaction in the Earth's atmosphere (e.g. Ferrari et al., 2001; Roesler et al., 2002). Over the years several models of this kind and/or based on other methods were proposed (Schraube et al., 2000; Ferrari et al., 2001; Roesler et al., 2002; Lewis et al., 2005; Takada et al., 2007; Matthiä et al., 2008; Sato et al., 2008; Latocha et al., 2009; Mertens et al., 2013; Matthiä et al., 2014; Mishev et al., 2014a; Wilson et al., 2014; Copeland, 2017), and a reasonable agreement between several models was achieved (Bottollier-Depois et al., 2009). While the models agree well between each other, it was recently shown that differences in input spectral and angular characteristics of SEPs can lead to significant, up to an order of magnitude, differences of the computed radiation exposure(s) (for details see Bütikofer \& Flückiger, 2013, 2015).

Herein, we employed a recent numerical model for computation of the effective and/or ambient dose equivalent at flight altitudes. The model is based on pre-computed yield functions (see details in Mishev \& Usoskin, 2015). The yield function represents the effective dose produced by a monoenergetic unit flux of primary CR particle, which enters in the atmosphere. It is obtained on the basis of high-statistics Monte Carlo simulations. The effective dose rate at a given atmospheric depth $h$ induced by a primary CR particle is computed by convolution of the yield function with a corresponding primary CR particle spectrum:

$$
E(h)=\sum_{i} \int_{T_{\text {cut }}\left(P_{c}\right)}^{\infty} J_{i}(T) Y_{i}(T, h) \mathrm{d} T
$$

where $J_{i}(T)$ is the differential energy spectrum of the primary CR arriving at the top of the atmosphere for $i$-th component (proton or $\alpha$-particle) and $Y_{i}$ is the effective dose yield function for this type of particles. The integration is over the kinetic energy $T$ above $T$ cut $(P c)$, which is defined by the local cut-off rigidity $P_{\mathrm{c}}$ for a nuclei of type $i$ $T_{\text {cut }, \mathrm{i}}=\sqrt{\left(\frac{Z_{i}}{A_{i}}\right)^{2} P_{c}^{2}+E_{0}^{2}}-E_{0}$, where $E 0=0.938 \mathrm{GeV} / \mathrm{c}^{2}$ is the proton's rest mass.

Accordingly, the effective dose yield function $Y i$ is defined as:

$$
Y_{i}(T, h)=\sum_{j} \int_{T^{*}} F_{i, j}\left(h, T, T^{*}, \theta, \varphi\right) C_{j}\left(T^{*}\right) \mathrm{d} T^{*}
$$

where $C_{j}\left(T^{*}\right)$ is the coefficient converting the fluence of secondary particles of type $j$ (neutron, proton, $\gamma, e^{-}, e^{+}, \mu^{-}, \mu^{+}$ $\left.\pi^{-}, \pi^{+}\right)$with energy $T^{*}$ to the effective dose, $F_{i, j}\left(h, T, T^{*}, \theta\right.$, $\varphi)$ is the fluence of secondary particles of type $j$, produced by a primary particle of type $i$ (proton or $\alpha$-particle) with a given primary energy $T$ arriving at the top of the atmosphere from zenith angle $\theta$ and azimuth angle $\varphi$. The conversion coefficients $C_{j}\left(T^{*}\right)$ are considered according to Pelliccioni (2000); Petoussi-Henss et al. (2010). Similar expressions are used for the ambient dose equivalent, the details and look-up tables are given in Mishev \& Usoskin (2015).

Herein, for computations of the ambient dose equivalent (Fig. 3) or effective dose we employ the force field model of GCR spectrum (Gleeson \& Axford, 1968; Caballero-Lopez \& Moraal, 2004; Usoskin et al., 2005), where the solar modulation parameter was considered according to Usoskin et al. (2011, 


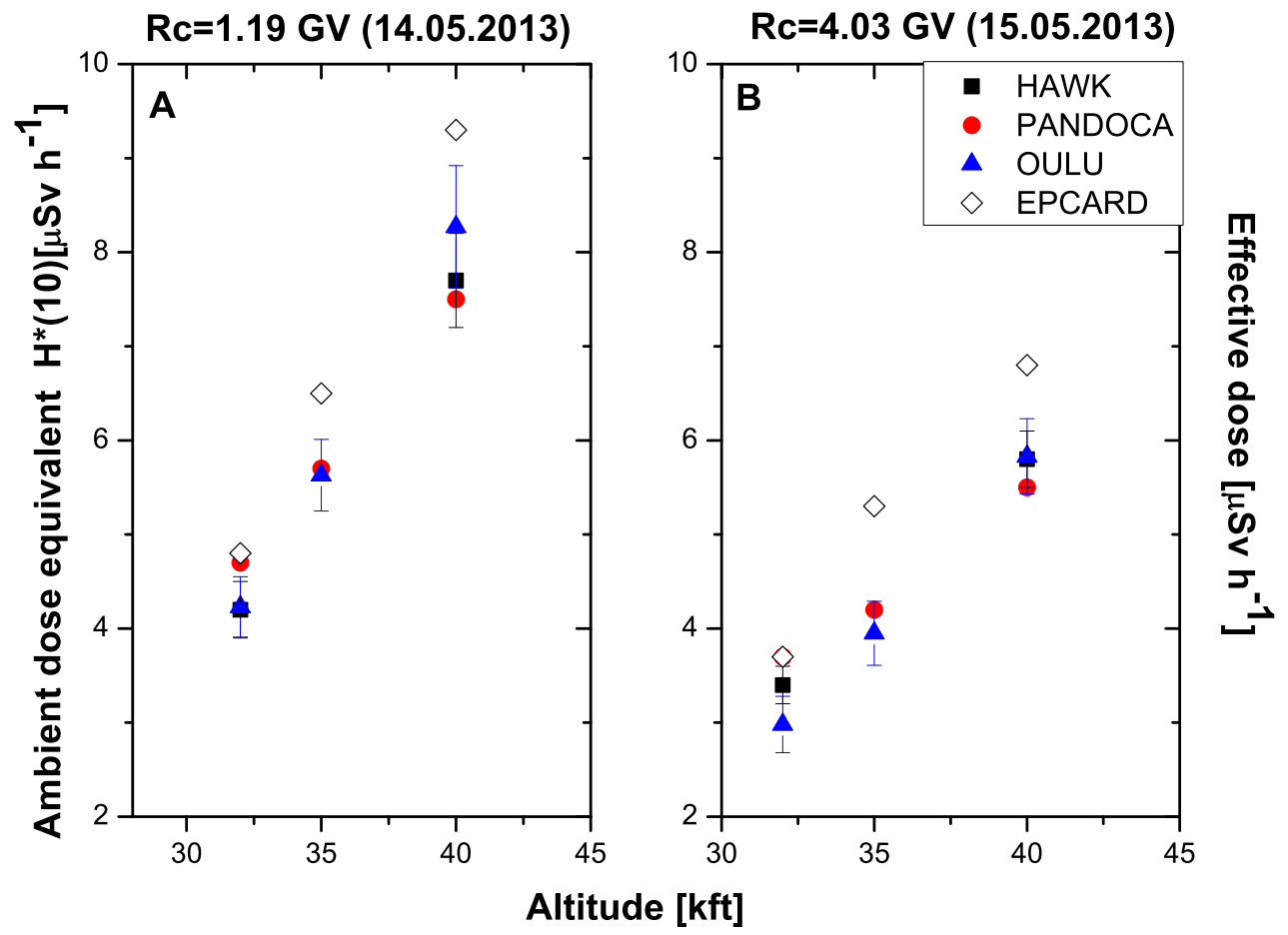

Fig. 3. Ambient dose equivalent $H^{*}(10)$ due to GCR at two geographic locations (panel a South Norway; panel b South Germany) at various altitudes computed with Oulu model compared with recent measurements and two models. The cut-off rigidity and date are given on the top of the figure. The measurements with HAWK environmental radiation monitoring system are adopted from (Meier et al., 2016), the estimations with PANDOCA model are interpolated from (Matthiä et al., 2014). Note that the EPCARD results are in effective dose.

2017). We consider a realistic mass composition of GCRs with the nucleonic ratio of heavier particles including $\alpha$-particles to protons in the interstellar medium as 0.3 similarly to (Mishev \& Velinov, 2011; Kovaltsov et al., 2012). The local interstellar spectrum was taken according to Burger et al. (2000).

Applied for computation of the radiation exposure due to GCR, the model demonstrated a very good agreement with the reference data (Menzel, 2010) and other model (Mertens et al., 2013), the details are given elsewhere by Mishev \& Usoskin (2015). In this study, the model is compared with recent measurements and two other widely used models at two different locations and three altitudes (Fig. 3) (Schraube et al., 2000; Matthiä et al., 2014; Meier et al., 2016). One can see the good agreement, specifically between Oulu and PANDOCA models, with recent experimental data. Note, that the EPCARD model provides effective dose rate, while the other models are applied here for ambient dose equivalent computation for the given altitude and location. However, a good agreement is observed, which is consistent with (Mertens et al., 2013; Mishev \& Usoskin, 2015) results.

\section{Assessment of effective dose at flight altitude}

Assessment of the effective dose at a flight altitude during GLE events is challenging, since events possess different features, viz. energy spectrum, duration and time evolution
(Gopalswamy et al., 2012; Moraal \& McCracken, 2012) and also because of the large diversity of secondaries at the flight altitude (e.g. Spurny et al., 1996). Therefore it is necessary to study each GLE event individually. For correct computations, it is necessary to derive precise spectral and angular characteristics of GLE particles in order to compute the effective dose rate (Eq. (1)). In general, this is possible using the NM data, but it requires such time consuming operations as computations of asymptotic cones, modelling of the NM response and fulfilling an optimization (e.g. Shea \& Smart, 1982; Humble et al., 1991; Cramp et al., 1997; Bombardieri et al., 2006; Vashenyuk et al., 2006; Mishev \& Usoskin, 2016; Mishev et al., 2017). Moreover, different sets of the derived spectra result in considerably different assessments of the dose rate (Bütikofer \& Flückiger, 2013, 2015).

Here we computed the effective dose rate at the altitude of $35 \mathrm{kft}(\approx 11 \mathrm{~km}$ a.s.l.) during GLE events, using rigidity spectra derived from the NM data (Debrunner et al., 1984; Lockwood et al., 1990a; Humble et al., 1991; Smart et al., 1993; Cramp et al., 1997b; Lovell et al., 1998; Deeley et al., 2002; Bombardieri et al., 2006, 2007, 2008; Bütikofer et al., 2009; Matthiä et al., 2009b; Vashenyuk et al., 2011; Bieber et al., 2013; Mishev et al., 2014b, 2017; Plainaki et al., 2014; Kravtsova \& Sdobnov, 2016; Mishev \& Usoskin, 2016; Kocharov et al., 2017; Mishev et al., 2018).

We conservatively assumed an isotropic distribution of the GLE particles similarly to Copeland et al. (2008) in order to assess the maximum radiation exposure. An important anisotropy is observed in most of GLE events, particularly during 
Table 2. Assessed maximum effective dose rates at the altitude of $35 \mathrm{kft}$ a.s.l. in a region with $P_{c}<1 \mathrm{GV}$ during GLE events. The columns depict: 1 - GLE number; 2 - GLE date; 3 - maximum effective dose rate due to solar protons; 4 - maximum NM increase with corresponding NM station; 5 - maximum NM increase at the sea level with the corresponding NM station. The station abbreviations and characteristics are given in Table 3. The footnotes in column 1 depict the corresponding bibliographic sources of the derived SEP spectra.

\begin{tabular}{|c|c|c|c|c|}
\hline GLE & Date & Max. $E \mu \mathrm{Sv}$ h-1 & Max. NM increase, $\%$ & Max. NM increase (sea level) \% \\
\hline $5^{1}$ & 23.02 .1956 & $\approx 900-2980$ & 5115 (LEED) & 5115 (LEED) \\
\hline $8^{1,2}$ & 04.05 .1960 & 52 & 331 (SLPM) & 261 (CHUR) \\
\hline $10^{1}$ & 12.11 .1960 & 12 & 156 (MTWS) & 108 (THUL) \\
\hline $13^{1}$ & 18.07 .1961 & 8 & 19 (THUL) & 19 (THUL) \\
\hline $16^{1}$ & 28.01 .1967 & 7 & 25.6 (VSTK) & 21 (WLKS) \\
\hline $19^{1}$ & 18.11.1969 & 5 & 32.4 (VSTK) & 26.6 (WLKS) \\
\hline $29^{1}$ & 24.09 .1977 & 2 & 10.9 (SOPO) & 8.9 (SNAE) \\
\hline $30^{1,3}$ & 22.11 .1977 & $10-45$ & $55.3(\mathrm{SOPO})$ & 32.8 (GSBY) \\
\hline $31^{1,3,4}$ & 07.05 .1978 & $21-35$ & 214 (KERG) & 214 (KERG) \\
\hline $32^{1}$ & 23.09 .1978 & 1.5 & $13.2(\mathrm{SOPO})$ & 10.7 (SNAE) \\
\hline $38^{1,5}$ & 07.12 .1982 & $7-15$ & 56 (KERG) & 56 (KERG) \\
\hline $39^{1}$ & 16.02 .1984 & 100 & 212 (SOPO) & 98 (GSBY) \\
\hline $45^{1,8}$ & 24.10 .1989 & $90-123$ & 205 (SOPO) & 120 (TERA) \\
\hline $47^{1}$ & 21.05 .1990 & 4.8 & 23.6 (THUL) & 23.6 (THUL) \\
\hline $48^{1}$ & 24.05 .1990 & 8.3 & 52.3 (MTWS) & 131.3 (INVK) \\
\hline $51^{1}$ & 11.06 .1991 & 3.4 & 9.2 (SOPO) & 8.0 (MWSN) \\
\hline $52^{1,9}$ & 15.06 .1991 & $6-8$ & 48 (SOPO) & 27.5 (KERG) \\
\hline $55^{1,10}$ & 06.11 .1997 & $3.5-9$ & 16.6 (SOPO) & 11.4 (OULU) \\
\hline $59^{1,11,12}$ & 14.07 .2000 & $18-43$ & 57.8 (SOPO) & 40.4 (THUL) \\
\hline $60^{1,13}$ & 15.04 .2001 & $20-47.5$ & 225 (SOPO) & 117.8 (NAIN) \\
\hline $61^{1}$ & 18.04 .2001 & 2.6 & 24.1 (SOPO) & 18.3 (SNAE) \\
\hline $65^{1}$ & 28.10 .2003 & 14.5 & 24.7 (MCMD) & 24.7 (MCMD) \\
\hline $67^{1,14}$ & 02.11 .2003 & $11-24$ & 36 (SOPO) & 21.9 (MCMD) \\
\hline $69^{1,15,16,17}$ & 20.01 .2005 & $265-2910$ & 4808 (SOPO) & 2649 (TERA) \\
\hline
\end{tabular}

${ }^{1}$ (Vashenyuk et al., 2011); ${ }^{2}$ (Deeley et al., 2002); ${ }^{3}$ (Debrunner et al., 1984); ${ }^{4}$ (Lockwood et al., 1990a); ${ }^{5}$ (Cramp et al., $\left.1997 b\right) ;{ }^{6}$ (Humble et al., 1991); ${ }^{7}$ (Cramp et al., 1997); ${ }^{8}$ (Lovell et al., 1998); ${ }^{9}$ (Smart et al., 1993); ${ }^{10}$ (Kravtsova and Sdobnov, 2016); ${ }^{11}$ (Bombardieri et al., 2006); ${ }^{12}$ (Mishev and Usoskin, 2016); ${ }^{13}$ (Bombardieri et al., 2007); ${ }^{14}$ (Kocharov et al., 2017); ${ }^{15}$ (Bieber et al., 2013); ${ }^{16}$ (Bütikofer et al., 2009); ${ }^{17}$ (Bombardieri et al., 2008); ${ }^{18}$ (Matthiä et al., 2009b); ${ }^{19}$ (Plainaki et al., 2014); ${ }^{20}$ (Mishev et al., 2014b, 2017 ); ${ }^{21}$ (Mishev et al., 2018).

the event onset and initial phase (e.g. Cramp et al., 1997; Bombardieri et al., 2008; Bütikofer et al., 2009). The anisotropy reveals nonsymmetric solar proton flux over the globe. Therefore, the anisotropy effects are important specifically during the event onset and lead to non-uniform distribution of the exposure (e.g. Bütikofer et al., 2008; Matthiä et al., 2009a, b; Velinov et al., 2013; Mishev \& Velinov, 2015, 2018). The explicit consideration of the anisotropy would result on underestimation of the effective dose rate compared to an isotropic distribution. In addition, the effective dose rate rapidly decreases at regions with higher cut-off rigidity because of considerably softer spectrum of SEPs compared to GCRs. Therefore, in order to provide a conservative approach, we performed all the computations in a region with the geomagnetic cut-off rigidity $P_{\mathrm{c}}<1 \mathrm{GV}$, where the expected exposure is maximal.
Hence, we computed the maximum effective dose rate dueto GLE particles, as shown in Table 2. In cases when several spectral reconstructions of GLE particles are available for a given event, resulting in considerably different effective dose estimates (more than 30-40\%), we presented an effective dose range (e.g., for GLEs 30, 42, 45, 69). In this study we considered only the maximum radiation exposure in order to provide a conservative approach. Therefore, mainly the prompt hard component of the GLE particles was taken into account, since it results in the maximum radiation exposure, while the soft component will be studied in a separate work.

The computed peak effective dose rate due to energetic solar protons during 34 GLEs out of 72 registered, yields 47 different sets of exposure assessments. In general, the effective dose rate varies from several $\mu \mathrm{Sv} \mathrm{h}{ }^{-1}$ which is comparable 


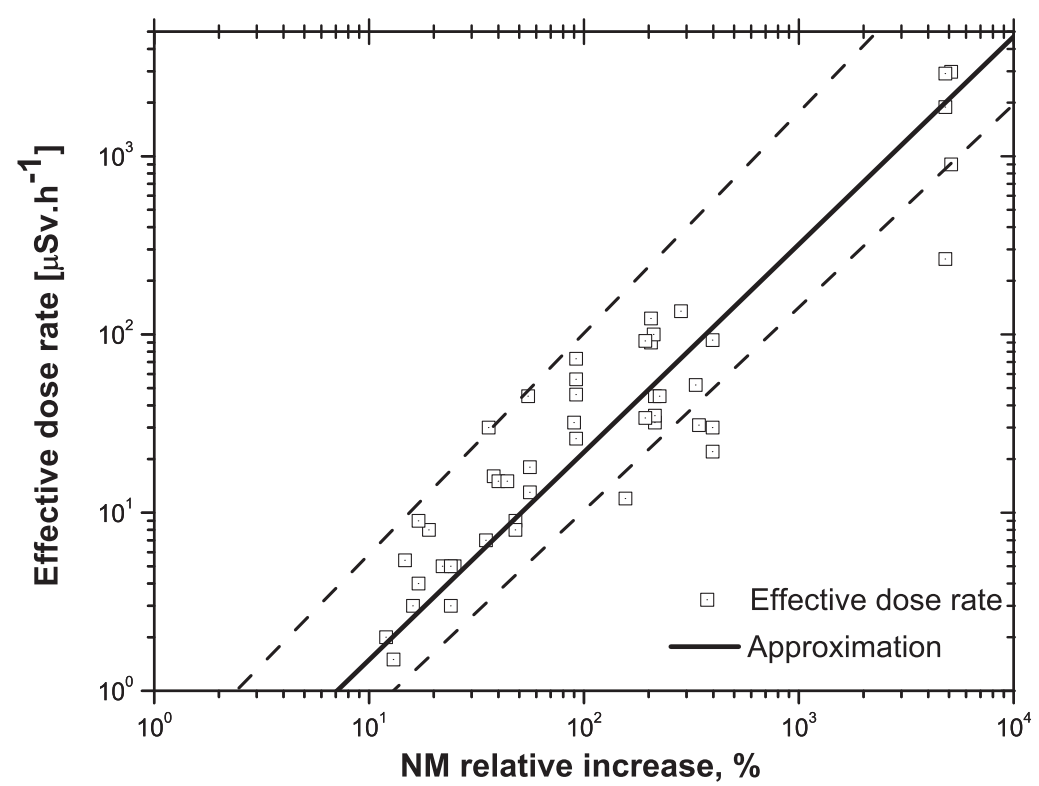

Fig. 4. The effective dose rate due to GLE particles, computed by the Oulu model, versus the maximum NM increase, along with the best-fit power-law functions (solid line) and its $95 \%$ confidence level (dashed lines).

Table 3. NM stations with maximum increase during GLE events used for analysis in this study. Cross (null) in the last column depict NMs active station (closed).

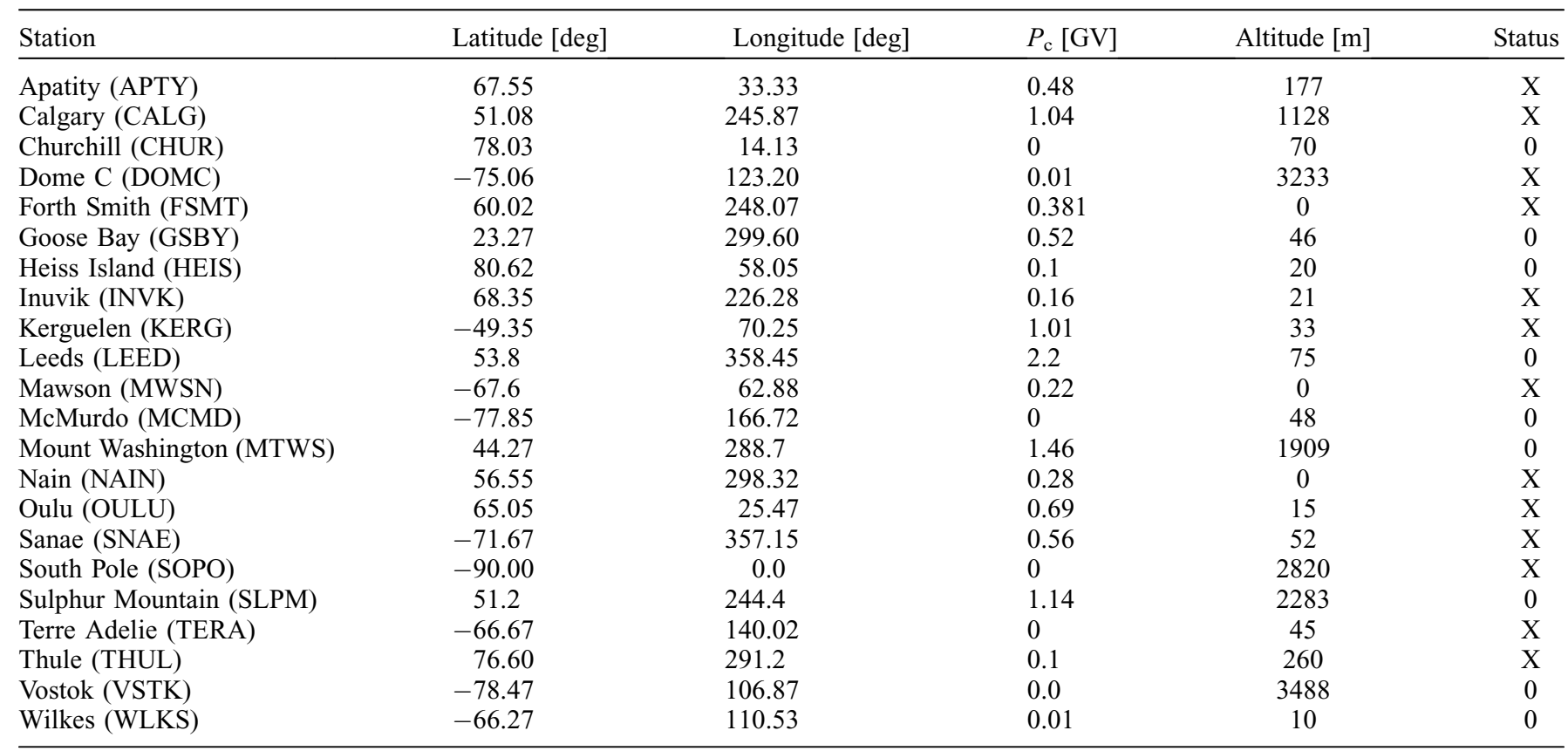

with the background GCR contribution (e.g., GLE 19, GLE 25, GLE 51) to tens or hundreds $\mu \mathrm{Sv} \mathrm{h}^{-1}$ (e.g. GLE 39, GLE 44, GLE 45). For the two strongest GLE events (GLE 5 and GLE $69)$, the dose appears in the range $1-2.5 \mathrm{mSv} \mathrm{h}^{-1}$. Subsequently, we compared the derived maximum effective dose rates with the maximum NM count rate increases for the corresponding event, i.e. constructing a distribution: maximum effective dose rate - peak NM increase during the event. We found a highly significant correlation (the Pearson's linear correlation coefficient of about 0.84 , with high significance level, namely $p^{-}$value $\ll 0.01$ ), between the maximum effective dose rate and peak NM count rate increase (Fig. 4). One can see that the proposed fit (Eq. (3)) encompasses, within the $95 \%$ confidence level, most of the data points. 
Table 4. Comparison of computed effective dose rates during several GLEs using Oulu and PANDOCA models. Columns 1 and 2 depict the GLE number and data, columns 3 and 4 depict the peak effective dose rate computed with Oulu and PANDOCA model, respectively, column 5 the relative difference between the models.

\begin{tabular}{lcccc}
\hline & & \multicolumn{2}{c}{${\mathrm{E} \mu \mathrm{Sv} \mathrm{h}^{-1}}$} & \\
\cline { 3 - 4 } GLE & Date & Oulu & PANDOCA & $\begin{array}{c}\text { Relative difference } \\
\%\end{array}$ \\
\hline 42 & 29.09 .1989 & 93 & $95-110$ & $1.8-15$ \\
69 & 20.05 .2005 & 1886 & 1800 & 4.6 \\
70 & 13.12 .2006 & 41 & $35-45$ & 9 \\
\hline
\end{tabular}

Table 5. Parameters of the best fit (Eq. (3)) of the distribution of the computed maximum effective rate due to GLE particles at the altitude of $35 \mathrm{kft}$ a.s.1 with the maximum NM increase during major GLEs (Table 2). Columns 3 and 4 depict the $95 \%$ confidence level of the parameters.

\begin{tabular}{lrrr}
\hline Parameter & \multicolumn{3}{c}{$95 \%$ confidence limits } \\
\hline$a$ & -2.2852 & -2.92556 & -1.6447 \\
$b$ & 1.1661 & 1.0905 & 1.2416 \\
\hline
\end{tabular}

NM count rate increase can be used as a convenient proxy for an assessment of the maximum effective dose at a flight altitude during a GLE event due to high energy SEPs.

A comparison of computed effective dose rates during several GLEs using Oulu Mishev \& Usoskin (2015) and PANDOCA (Matthiä et al., 2014) models is performed (see for details Table 4). Note, that here we assume the same GLE spectra (Matthiä et al., 2009a, b) in order to minimize the differences and avoid discrepancy due to different spectral reconstruction (e.g. Bütikofer \& Flückiger, 2013, 2015). One can see that an agreement within about $10 \%$ is achieved, the small difference is most likely due to anisotropy effects, GCR parametrization and/or model features as discussed in (Bottollier-Depois et al., 2009).

About $65 \%$ of the maximum NM increases are registered by low rigidity high-altitude stations (Table 2). However, several of those stations are not active (MTWS, SLPM and VSTK). Thus, only SOPO/B and DOMC/B, both stations located in South hemisphere, are low rigidity high-altitude NMs (Fig. 1). In order to provide convenient and effective proxy suitable for operational purposes and to avoid usage of a single NM, which may lead to a bias in a case of highly anisotropic events (Fig. 2), we performed a similar study but for sea level NMs (Table 2). We derived similar correlation as in the previous case, with the same quality. However, considering only sea level NMs, the fit of the distribution leads to more conservative assessment of the effective dose during GLEs (see below). Considering only the sea level NMs, the assessed effective dose is about $50 \%$ greater than the previous case i.e. a more conservative approach is proposed. In addition, it is more convenient for operational purposes, due to the large number of sea level uniformly distributed stations.

The distribution shown in Fig. 4 was fitted by the equation:

$$
E(x)=\exp (a+b \ln (x))
$$

where $E(x)$ is the maximum effective dose rate at the altitude of $35 \mathrm{kft}$ a.s.l. due to SEPs, $x$ corresponds to peak NM count increase in \% during the event, $a$ and $b$ are the fitted parameters, the details are given in Table 5. Note, that the GCR contribution to the exposure is routinely computed with model(s), explicitly considering the solar activity, and shall be superposed with Equation (3) calculations.

\section{Conclusions}

In this work a number of GLE events have been studied, where the necessary information was available. On the basis of the SEP rigidity spectra derived from NM records, the maximum effective dose rates at the aviation altitude of $35 \mathrm{kft}$ during these events was calculated using a recently proposed model. A highly significant correlation between the maximum effective dose rate due to solar protons and the peak NM count rate increase was found. We propose to use the NM count rate increase as a proxy to assess the effective dose at a flight altitude. Thismakes it possible to obtain a quick estimate of the effective dose rate due to SEPs during GLE events on the basis of records from the global NM network, specifically the low cut-off rigidity stations (see Table 1 and Fig. 2).

Note, that in most cases, the GLE effective dose rate profile, consists of rapid rising at the event onset at initial phase, followed by a decay. Therefore, the peak effective dose rate is due mostly to the hard - prompt component. In addition, the most energetic solar protons from the prompt component arrive in the vicinity of Earth before the bulk of SEPs. Therefore, approximations of the dose rates for operational purposes can be achieved instantly (cf. Latocha et al., 2009). Hence, the global NM network can be used to assess an important space weather effect, namely the radiation exposure of aircrew due to high energy particles of solar origin.

Acknowledgements. This work was supported by the Academy of Finland (project 272157, Center of Excellence ReSoLVE) and VarSITI/SCOSTEP project. The authors are warmly thankful to Askar Ibragimov for the GLE database support. The authors acknowledge all the researchers, NM station managers and colleagues who collected the GLE records and performed data analysis. The authors would like to thank the anonymous reviewers for their comments and suggestions that have contributed to improve this paper. The editor thanks Kyle Copeland and two anonymous referees for their assistance in evaluating this paper.

\section{References}

Adriani O, Barbarino G, Bazilevskaya G, Bellotti R, Boezio M, et al. 2016. Measurements of cosmic-ray Hydrogen and Helium isotopes with the PAMELA experiment. ApJ 818(1). DOI: 10.3847/0004-637X/818/1/68

Aguilar M, Alcaraz J, Allaby J, Alpat B, Ambrosi G, et al. 2010. Relative composition and energy spectra of light nuclei in cosmic rays: Results from AMS-01 ApJ 724 (1): 329-340. DOI: 10.1088/ 0004-637X/724/1/329. 
Baker D. 1998. What is space weather? Adv Space Res 22 (1): 7-16. Bazilevskaya GA, Usoskin IG, Flückiger E, Harrison R, Desorgher L, et al. 2008. Cosmic Ray induced ion production in the atmosphere. Space Sci Rev 137: 149-173. DOI: 10.1007/s11214-008-9339-y.

Bieber J, Clem J, Evenson P, Pyle R, Sáiz A, Ruffolo D. 2013. Giant ground level enhancement of relativistic solar protons on 2005 January 20. I. Spaceship earth observations. ApJ 771 (2): 92. DOI: 10.1088/0004-637X/771/2/92.

Bieber J, Evenson P. 1995. Spaceship Earth - An Optimized Network of Neutron Monitors. In Proc. of 24th ICRC Rome, Italy, 28 August - 8 September 1995, 4: 1316-1319.

Bombardieri D, Duldig M, Humble J, Michael K. 2008. An improved model for relativistic solar proton acceleration applied to the 2005 January 20 and earlier events $A p J 682$ (2): 1315-1327. DOI: $10.1086 / 589494$.

Bombardieri D, Duldig M, Michael K, Humble J. 2006. Relativistic proton production during the 2000 July 14 solar event: The case for multiple source mechanisms. ApJ 644 (1): 565-574. DOI: 10.1086/501519.

Bombardieri D, Michael K, Duldig M, Humble J. 2007. Relativistic proton production during the 2001 April 15 solar event. ApJ $\mathbf{6 6 5}$ (1): 813-823. DOI: 10.1086/519514.

Bottollier-Depois J, Beck P, Bennett B, Bennett L, Bütikofer R, et al. 2009. Comparison of codes assessing galactic cosmic radiation exposure of aircraft crew. Radiat Prot Dosim 136 (4): 317-323. DOI: $10.1093 / \mathrm{rpd} / \mathrm{ncp} 159$.

Burger R, Potgieter M, Heber B. 2000. Rigidity dependence of cosmic ray proton latitudinal gradients measured by the Ulysses spacecraft: Implication for the diffusion tensor. J Geophys Res, 105, 27447-27455. DOI: 10.1029/2000JA000153.

Bütikofer R, Flückiger E. 2013. Differences in published characteristics of GLE60 and their consequences on computed radiation dose rates along selected flight paths. J Phys Conf Ser, 409(1), 012166. DOI: 10.1088/1742-6596/409/1/012166.

Bütikofer R, Flückiger E. 2015. What are the causes for the spread of GLE parameters deduced from NM data? J Phys: Conf Ser, 632(1), 012053, DOI: 10.1088/17426596/632/1/012053.

Bütikofer R, Flückiger E, Desorgher L, Moser M. 2008. The extreme solar cosmic ray particle event on 20 January 2005 and its influence on the radiation dose rate at aircraft altitude. Sci Total Environ, 391(2-3), 177-183.

Bütikofer R, Flückiger E, Desorgher L, Moser M, Pirard B. 2009. The solar cosmic ray ground-level enhancements on 20 January 2005 and 13 December 2006. Adv Space Res 43 (4): 499-503.

Caballero-Lopez R, Moraal H. 2004. Limitations of the force field equation to describe cosmic ray modulation. J Geophys Res 109: A01101, DOI: 10.1029/2003JA010098.

Carmichael H. 1968. Cosmic rays (instruments). In Ann. IQSY, vol. 1, Minnis CM (Ed.), MIT Press, Cambridge, MA, pp. 178-197.

Cliver E, Kahler S, Reames D. 2004. Coronal Shocks and Solar Energetic Proton Events. ApJ 605: 902-910, 10.1086/382651.

Copeland K. 2017. Cari-7A: Development and validation. Radiat Prot Dosim 175 (4): 419-431. DOI: 10.1093/rpd/ncw369.

Copeland K, Sauer H, Duke F, Friedberg W. 2008. Cosmic radiation exposure of aircraft occupants on simulated high-latitude flights during solar proton events from 1 January 1986 through 1 January 2008. Adv Space Res 42 (6): 1008-1029. DOI: 10.1016/ j.asr.2008.03.001.

Cramp J, Duldig M, Flückiger E, Humble J, Shea M, Smart D. 1997. The October 22, 1989, solar cosmic enhancement: An analysis the anisotropy spectral characteristics. $J$ Geophys Res, 102, 24237-24248. DOI: 10.1029/97JA01947.
Cramp J, Duldig M, Humble J. 1997. The effect of a distorted interplanetary magnetic field configuration on the December 7-8, 1982, ground level enhancement.J Geophys Res A: Space Phys 102 (A3): 4919-4925.

Debrunner H, Flückiger E, Gradel H, Lockwood J, Mcguire R. 1988. Observations related to the acceleration, injection, and interplanetary propagation of energetic protons during the solar cosmic ray event on February 16, 1984. J Geophys Res 93 (A7): 7206-7216. DOI: 10.1029/JA093iA07p07206.

Debrunner H, Flückiger E, Lockwood J, McGuire R. 1984. Comparison of the solar cosmic ray events on May 7, 1978, and November 22, 1977. J Geophys Res 89 (A2): 769-774.

Deeley K, Duldig M, Humble J. 2002. Re-analysis of the cosmic ray ground level enhancement of 4 May 1960. Adv Space Res 30 (4) 1049-1052. DOI: 10.1016/S0273-1177(02)00495-7.

Desai M, Giacalone J. 2016. Large gradual solar energetic particle events. Living Rev Sol Phys 13 (1): 3. DOI: 10.1007/s41116-0160002-5.

Desorgher L, Flückiger E, Gurtner M, Moser M, Bütikofer R. 2005. A Geant 4 code for computing the interaction of cosmic rays with the earth's atmosphere. Int J Mod Phys A 20 (A11): 6802-6804, 10.1142/S0217751X05030132.

Dorman L. 2004. Cosmic Rays in the Earth's Atmosphere and Underground, Kluwer Academic Publishers, Dordrecht. ISBN 1-4020-2071-6.

Dorman L. 2006. Cosmic Ray Interactions, Propagation, and Acceleration in Space Plasmas. Astrophysics and Space Science Library 339, Springer, Dordrecht, ISBN 13-978-1-40205100-5

Eastwood J, Nakamura R, Turc L, Mejnertsen L, Hesse M. 2017. The Scientific Foundations of Forecasting Magnetospheric Space Weather. Space Sci Rev 212(3-4): 1221-1252. DOI: 10.1007/ s11214-017-0399-8.

EURATOM. 2013. Council directive 2013/59/EURATOM of 5 December 2013 laying down basic safety standards for protection against the dangers arising from exposure to ionising radiation, and repealing Directives 89/618/Euratom, 90/641/Euratom, 96/29/ Euratom, 97/43/Euratom and 2003/122/Euratom. OJEC L13.

Ferrari A, Pelliccioni M, Rancati T. 2001. Calculation of the radiation environment caused by galactic cosmic rays for determining air crew exposure. Radiat Prot Dosim 93 (2): 101-114. DOI: 10.1093/oxfordjournals.rpd.a006418.

Gaisser TK, Stanev T. 2010. Cosmic rays. In K. Nakamura et al., Review of particle physics. $J$ Phys $G, 37,269-275$.

Ganushkina N, Jaynes A, Liemohn M. 2017. Space weather effects produced by the ring current particles. Space Sci Rev, 212(3-4), 1315-1344. DOI: 10.1007/s11214-017-0412-2.

Gil A, Usoskin I, Kovaltsov G, Mishev A, Corti C, Bindi V. 2015. Can we properly model the neutron monitor count rate? $J$ Geophys Res, 120, 7172-7178. DOI: 10.1002/2015JA021654.

Gleeson L, Axford W. 1968. Solar modulation of galactic cosmic rays. ApJ 154: 1011-1026.

Gopalswamy N, Xie H, Yashiro S, Akiyama S, Mäkelä P, Usoskin I. 2012. Properties of ground level enhancement events and the associated solar eruptions during solar cycle 23. Space Sci Rev 171 (1-4): 23-60.

Grieder P. 2001. Cosmic rays at Earth researcher's reference manual and data book Elsevier Science: Amsterdam. ISBN 978-0-44450710-5.

Hatton C. 1971. The neutron monitor. In Progress in Elementary Particle and Cosmic-ray Physics X, chap. 1, Elsevier Science: Amsterdam. ISBN 978-0-444-50710-5. 
Humble J, Duldig M, Smart D, Shea M. 1991. Detection of 0.515 GEV solar protons on 29 September 1989 at Australian stations. Geophys Res Lett, 18 (4): 737-740.

ICRP. 2007. ICRP Publication 103: The 2007 Recommendations of the International Commission on Radiological Protection. Ann ICRP 37 (2-4): 2007.

Klein K-L, Dalla S. 2017. Acceleration and propagation of solar energetic particles. Space Sci Rev 212 (3-4): 1107-1136. DOI: 10.1007/s11214-017-0382-4.

Kocharov L, Pohjolainen S, Mishev A, Reiner MJ, Lee J, et al. 2017. Investigating the origins of two extreme solar particle events: Proton source profile and associated electromagnetic emissions. ApJ 839 (2): 79. DOI: 10.3847/1538-4357/aa6a13.

Koskinen H, Baker D, Balogh A, Gombosi T, Veronig A, von Steiger R. 2017. Achievements and challenges in the science of space weather. Space Sci Rev 212 (3-4): 1137-1157. DOI: 10.1007/ s11214-017-0390-4.

Kovaltsov G, Mishev A, Usoskin I. 2012. A new model of cosmogenic production of radiocarbon $14 \mathrm{C}$ in the atmosphere. Earth Planet. Sci. Lett. 337: 114-120. DOI: 10.1016/j.epsl.2012.05.036.

Kravtsova MV, Sdobnov VE. 2016. Ground level enhancement of cosmic rays on November 6, 1997: Spectra and anisotropy. JETP Lett 103 (1): 8-14. DOI: 10.1134/S0021364016010094.

Kudela K. 2016. On low energy cosmic rays and energetic particles near Earth. CAOSP 46 (1): 15-70.

Kudela K, Usoskin I. 2004. On magnetospheric transmissivity of cosmic rays. Czech J Phys 54 (2): 239-254.

Latocha M, Beck P, Rollet S. 2009. AVIDOS - a software package for European accredited aviation Dosimetry. Radiat Prot Dosim 136 (4): 286-290. DOI: 10.1093/rpd/ncp126.

Lewis B, Bennett L, Green A, Butler A, Desormeaux M, Kitching F, McCall M, Ellaschuk B, Pierre M. 2005. Aircrew dosimetry using the Predictive Code for Aircrew Radiation Exposure (PCAIRE). Radiat Prot Dosim 116 (1-4): 320-326.

Lilensten L, Bornarel J. 2009. Space weather, environment and societies. Springer: Dordrecht ISBN 978-1-4020-4332-1.

Lockwood J, Debrunner H, Flükiger E, Grädel H. 1990a. Proton energy spectra at the sun in the solar cosmic-ray events on 1978 May 7 and 1984 February 16. ApJ 355 (1): 287-294.

Lockwood JA, Debrunner H, Flükiger EO. 1990b. Indications for diffusive coronal shock acceleration of protons in selected solar cosmic ray events. J Geophys Res: Space Phys, 95 (A4), 4187-4201.

Lovell JL, Duldig ML, Humble JE. 1998. An extended analysis of the September 1989 cosmic ray ground level enhancement. $J$ Geophys Res: Space Phys, 103 (A10), 23733-23742. DOI: 10.1029/98JA02100.

Macmillan S, Maus S, Bondar T, Chambodut A, Golovkov V, et al. 2003. The 9th-Generation International Geomagnetic Reference Field. Geophys J Int 155 (3): 1051-1056. DOI: 10.1111/j.1365246X.2003.02102.x.

Matthiä D, Heber B, Reitz G, Meier M, Sihver L, Berger T, Herbst K. 2009a. Temporal and spatial evolution of the solar energetic particle event on 20 January 2005 and resulting radiation doses in aviation. J Geophys Res: Space Phys, 114(8): 8104.

Matthiä D, Heber B, Reitz G, Sihver L, Berger T, Meier M. 2009b. The ground level event 70 on December 13th, 2006 and related effective doses at aviation altitudes. Radiat Prot Dosim 136(4) 304-310.

Matthiä D, Meier M, Reitz G. 2014. Numerical calculation of the radiation exposure from galactic cosmic rays at aviation altitudes with the PANDOCA core model. Space Weather 12 (3): 161-171. DOI: $10.1002 / 2013$ SW001022.
Matthiä D, Sihver L, Meier M. 2008. Monte-Carlo calculations of particle fluences and neutron effective dose rates in the atmosphere. Radiat Prot Dosim 131 (2): 222-228. DOI: 10.1093/rpd/ncn130.

Mavromichalaki H, Papaioannou A, Plainaki C, Sarlanis C, Souvatzoglou G, et al. 2011. Applications and usage of the realtime Neutron Monitor Database. Adv Space Res, 47, 2210-2222. DOI: $10.1016 /$ j.asr.2010.02.019.

Meier M, Trompier F, Ambrozova I, Kubancak J, Matthi D, Ploc O, Santen N, Wirtz M. 2016. CONCORD: Comparison of cosmic radiation detectors in the radiation field at aviation altitudes. J Space Weather Space Clim, 6): A24. DOI: 10.1051/swsc/2016017.

Menzel H. 2010. The international commission on radiation units and measurements. J. ICRU 10 (2): 1-35.

Menzel H. 2010. The international commission on radiation units and measurements. J. ICRU 10 (2): 1-35.

Mertens C, Meier M, Brown S, Norman R, Xu X. 2013. NAIRAS aircraft radiation model development, dose climatology, and initial validation. Space Weather 11 (10): 603-635. DOI: 10.1002/ swe. 20100.

Mewaldt R. 2006. Solar energetic particle composition, energy spectra, and space weather. Space Sci Rev 124 (1-4): 303-316. DOI: $10.1007 / \mathrm{s} 11214-006-9091-0$.

Mishev A, Adibpour F, Usoskin I, Felsberger E. 2014. Computation of dose rate at flight altitudes during ground level enhancements no. 69, 70 and 71. Adv Space Res 55 (1): 354-362. DOI: 10.1016/ j.asr.2014.06.020.

Mishev A, Kocharov L, Usoskin I. 2014b. Analysis of the ground level enhancement on 17 May 2012 using data from the global neutron monitor network. J Geophys Res, 119, 670-679. DOI: 10.1002/2013JA019253.

Mishev A, Poluianov S, Usoskin I. 2017. Assessment of spectral and angular characteristics of sub-GLE events using the global neutron monitor network. J Space Weather Space Clim, 7, A28. DOI: $10.1051 /$ swsc/2017026.

Mishev A, Usoskin I. 2013. Computations of cosmic ray propagation in the Earth's atmosphere, towards a GLE analysis. J Phys Conf Ser, 409, 012152. DOI: 10.1088/1742-6596/409/1/012152.

Mishev A, Usoskin I. 2015. Numerical model for computation of effective and ambient dose equivalent at flight altitudes: Application for dose assessment during GLEs. J Space Weather Space Clim 5 (3): A10. DOI: 10.1051/swsc/2015011.

Mishev A, Usoskin I. 2016. Analysis of the ground level enhancements on 14 July 2000 and on 13 December 2006 using neutron monitor data. Sol Phys 291 (4): 1225-1239.

Mishev A, Usoskin I, Raukunen O, Paassilta M, Valtonen E, Kocharov L, Vainio R. 2018. First analysis of GLE 72 event on 10 September 2017: Spectral and anisotropy characteristics. Sol Phys, in press. DOI: 10.1007/s11207-018-1354-x.

Mishev A, Velinov P. 2011. Normalized Ionization Yield function for various nuclei obtained with full Monte-Carlo simulations. Adv Space Res 48 (1): 19-24.

Mishev A, Velinov P. 2015. Time evolution of ionization effect due to cosmic rays in terrestrial atmosphere during GLE 70. J Atmos Sol Terr Phys 129: 78-86.

Mishev A, Velinov P. 2018. Ion production and ionization effect in the atmosphere during the Bastille day GLE 59 due to high energy SEPs. Adv Space Res 61 (1): 316-325. DOI: 10.1016/ j.asr.2017.10.023.

Moraal H. 1976. Observations of the eleven-year cosmic-ray modulation cycle. Space Sci Rev 19 (6): 845-920.

Moraal H, Belov A, Clem J. 2000. Design and co-ordination of multi-station international neutron monitor networks. Space Sci $\operatorname{Rev} 93$ (1-2): 285-303. 
Moraal H, McCracken K. 2012. The time structure of ground level enhancements in solar cycle 23. Space Sci Rev 171 (1-4): 85-95.

Nevalainen J, Usoskin I, Mishev A. 2013. Eccentric dipole approximation of the geomagnetic field: Application to cosmic ray computations. Adv Space Res 52 (1): 22-29.

Pelliccioni M. 2000. Overview of fluence-to-effective dose and fluence-to-ambient dose equivalent conversion coefficients for high energy radiation calculated using the FLUKA Code. Radiat Prot Dosim 88 (4): 279-297.

Petoussi-Henss N, Bolch W, Eckerman K, Endo A, Hertel N, Hunt J, Pelliccioni M, Schlattl H, Zank1 M. 2010. Conversion Coefficients for Radiological Protection Quantities for External Radiation Exposures. Ann ICRP 40 (2-5): 1-257.

Plainaki C, Mavromichalaki H, Laurenza M, Gerontidou M, Kanellakopoulos A, Storini M. 2014. The ground-level enhancement of 2012 May 17: Derivation of solar proton event properties through the application of the NMBANGLE PPOLA model. ApJ 785 (2): 160. DOI: 10.1088/0004-637X/785/2/160.

Potgieter M. 2013. Solar modulation of cosmic rays. Living Rev Sol Phys, 10: 274. DOI: 10.12942/lrsp-2013-3.

Pulkkinen T. 2007. Space weather: Terrestrial perspective. Living Rev Sol Phys 4 (1): 1-60.

Reames D. 1999. Particle acceleration at the Sun and in the heliosphere. Space Sci Rev 90 (3-4): 413-491.

Reames D. 2013. The two sources of solar energetic particles. Space Sci Rev 175 (1-4): 53-92. DOI: 10.1007/s11214-013-9958-9.

Roesler S, Heinrich W, Schraube H. 2002. Monte Carlo calculation of the radiation field at aircraft altitudes. Radiat Prot Dosim 98 (4): 367-388.

Sato T, Kataoka R, Yasuda H, Yashiro S, Kuwabara T, Shiota D, Kubo Y. 2014. Air shower simulation for WASAVIES: Warning system for aviation exposure to solar energetic particles. Radiat Prot Dosim 161 (1-4): 274-278. DOI: 10.1093/rpd/nct332.

Sato T, Yasuda H, Niita K, Endo A, Sihver L. 2008. Development of PARMA: PHITS-based analytical radiation model in the atmosphere. Radiat Res, 170, 244-259.

Schraube H, Leuthold G, Heinrich W, Roesler S, Combecher D. 2000. European program package for the calculation of aviation route doses version 3.0. Tech. Rep. D-85758, National Research Center for Environment and Health Institute of Radiation Protection, Neuherberg, Germany.

Shea M, Smart D. 1982. Possible evidence for a rigidity-dependent release of relativistic protons from the solar corona. Space Sci Rev 32: 251-271.

Shea M, Smart D. 1990. A summary of major solar proton events. Sol Phys 127: 297-320.

Shea M, Smart D. 2000. Cosmic ray implications for human health. Space Sci Rev 93 (1-2): 187-205.

Shea M, Smart D. 2012. Space weather and the ground-level solar proton events of the 23rd solar cycle. Space Sci Rev 171: 161188.

Simpson J. 1957. Cosmic-radiation neutron intensity monitor. Annals of The International Geophysical Year 4: 351-373.

Simpson J, Fonger W, Treiman S. 1953. Cosmic Radiation Intensitytime variation and their origin. I. Neutron intensity variation method and meteorological factors. Phys Rev 90: 934-950.
Smart D, Shea M, Gentile L. 1993. The Relativistic Solar Proton Event of 15 June 1991. Proc. of 23th ICRC 19-30 Jul 1993, Calgary, Canada, Vol. 3: 59-62.

Spurny F, Votockova I, Bottollier-Depois J. 1996. Geographical influence on the radiation exposure of an aircrew on board a subsonic aircraft. Radioprotection 31 (2): 275-280.

Spurny F, Dachev T, Kudela K. 2003. Increase of onboard aircraft exposure level during a solar flare. Bezpecnost Jaderne Energie $11(3-4)$ : 103-107.

Stoker P. 1995. Relativistic solar proton events. Space Sci Rev 73 (3-4): 327-385. DOI: 10.1007/BF00751240.

Stoker P, Dorman L, Clem J. 2000. Neutron monitor design improvements. Space Sci Rev 93 (1-2): 361-380.

Takada M, Lewis B, Boudreau M, Anid H, Bennett L. 2007. Modeling of aircrew radiation exposure from galactic cosmic rays and solar particle events. Adv Geosci: Vol 8: Sol Terr, 233-243. DOI: $10.1142 / 6494-$ vol8

Tsyganenko N. 1989. A magnetospheric magnetic field model with a warped tail current sheet. Planet Space Sci 37 (1): 5-20.

Tuohino S, Ibragimov A, Usoskin I, Mishev A. 2018. Upgrade of GLE database: Assessment of effective dose rate at flight altitude. Adv Space Res 62 (2): 398-407. DOI: 10.1016/j.asr.2018. 04.021.

Usoskin I, Alanko-Huotari K, Kovaltsov G, Mursula K. 2005. Heliospheric modulation of cosmic rays: Monthly reconstruction for 1951-2004. J Geophys Res 110: A12108.

Usoskin I, Bazilevskaya G, Kovaltsov G. 2011. Solar modulation parameter for cosmic rays since 1936 reconstructed from groundbased neutron monitors and ionization chambers. $J$ Geophys Res 116: A02104. DOI: 10.1029/2010JA016105.

Usoskin I, Gil A, Kovaltsov G, Mishev A, Mikhailov V. 2017. Heliospheric modulation of cosmic rays during the neutron monitor era: Calibration using PAMELA data for 2006-2010. J Geophys Res: Space Phys 122 (4): 3875-3887. DOI: 10.1002/ 2016JA023819.

Usoskin IG, Desorgher L, Velinov P, Storini M, Flückiger E, Bütikofer R, Kovaltsov G. 2009. Ionization of the Earth's atmosphere by solar and galactic cosmic rays. Acta Geophys 57 (1): 88-101.

Vainio R, Desorgher L, Heynderickx D, Storini M, Flückiger E, et al. 2009. Dynamics of the Earth's particle radiation environment. Space Sci Rev 147 (3-4): 187-231.

Vashenyuk E, Balabin Y, Gvozdevsky B. 2011. Features of relativistic solar proton spectra derived from ground level enhancement events (GLE) modeling. Astrophys Space Sci Trans 7 (4): 459-463. DOI: 10.5194/astra-7-459-2011.

Vashenyuk E, Balabin Y, Perez-Peraza J, Gallegos-Cruz A, Miroshnichenko L. 2006. Some features of the sources of relativistic particles at the Sun in the solar cycles 21-23. $A d v$ Space Res 38 (3): 411-417.

Velinov P, Asenovski S, Kudela K, Lastovička J, Mateev L, Mishev A, Tonev P. 2013. Impact of cosmic rays and solar energetic particles on the Earth's ionosphere and atmosphere. J Space Weather Space Clim 3: A14. DOI: 10.1051/swsc/2013036.

Wilson J, Slaba T, Badavi F, Reddell B, Bahadori A. 2014. Advances in NASA radiation transport research: 3DHZETRN. Life Sci Space Res 2: 6-22. DOI: 10.1016/j.1ssr.2014.05.003.

Cite this article as: Mishev A, Tuohino S \& Usoskin I 2018. Neutron monitor count rate increase as a proxy for dose rate assessment at aviation altitudes during GLEs. J. Space Weather Space Clim. 8, A46. 